\title{
Contribución al conocimiento de las aves presentes en la costa rioplatense de los partidos de Quilmes y Avellaneda (Buenos Aires, Argentina)
}

\author{
E.S. Montalibet ${ }^{1}$, M.A. Sosa ${ }^{1}$, S. Rozadilla ${ }^{1,2}$, N. Lazarte $^{1}$ y F.M. Irazoqui ${ }^{1}$ \\ ${ }^{1}$ Facultad de Ciencias Naturales y Museo, Universidad Nacional de La Plata, Avenida 122 y 60 (1900) La Plata, Buenos Aires, Argentina. E- \\ mails: estrella.montalibet@gmail.com; alesosa_15@hotmail.com; nicolaslazarte7@gmail.com; facundopaleo@hotmail.com \\ ${ }^{2}$ Laboratorio de Anatomía Comparada y Evolución de los Vertebrados, Museo Argentino de Ciencias Naturales "Bernardino Rivadavia", Av. \\ Ángel Gallardo 470, C1405DJR Buenos Aires, Argentina. E-mail: sebastianrozadilla@gmail.com
}

\begin{abstract}
RESUMEN. En este trabajo se presentan nuevos registros de aves para la costa rioplatense de los partidos de Quilmes y Avellaneda (Provincia de Buenos Aires, Argentina). La zona estudiada incluye dos áreas protegidas: Reserva Natural Municipal Los Sauces y el Parque Natural y Reserva Ecológica Municipal Selva Marginal Quilmeña, como también sectores sin categoría de conservación, abarcando en conjunto más de 300 hectáreas. Las observaciones se realizaron tanto por el Club de Observadores de Aves de Bernal como por estudiantes de la Facultad de Ciencias Naturales y Museo (Universidad Nacional de la Plata). Se adiciona un total de 31 especies nuevas en relación a trabajos anteriores, pertenecientes a las familias Tinamidae (1), Falconidae (1), Anatidae (2), Ardeidae (1), Rallidae (3), Strigidae (2), Cuculidae (1), Alcedinidae (1), Furnariidae (7), Sturnidae (1), Motacillidae (1), Thraupidae (2), Tityridae (1), Tyrannidae (5), Icteridae (2). Destacan los registros de Spartonoica maluroides (Espartillero enano) y Asthenes hudsoni (Espartillero pampeano), ambas catalogadas como vulnerables, además de varias especies categorizadas como escasas y sensibles frente a disturbios antropogénicos. También se ha observado un ejemplar de Icterus croconotus (Matico), una especie natural de la ecorregión del Chaco Húmedo. Cabe señalar el registro de Acridotheres cristatellus (Estornino crestado), ave introducida en nuestro país, cuyo impacto en las especies nativas tanto de aves como de otros animales es desconocido. Con esta contribución, se adicionan registros con valor conservacionista y el número de especies observables pasa de 124 a 155 para éste área, que se encuentra bajo un importante impacto antrópico y es considerado un posible corredor biológico entre las reservas Costanera Sur y Punta Lara.
\end{abstract}

Palabras clave: Región rioplatense, corredor biótico, biodiversidad, Aves, Áreas Protegidas.

\begin{abstract}
Contribution to the knowledge of the birds of Quilmes and Avellaneda (Buenos Aires, Argentina). On this paper new bird registers are provided on the Quilmes and Avellaneda (Buenos Aires province, Argentina) district's river coast. The studied region includes two preserved areas: Municipal Natural Reserve Los Sauces and Natural Park and Municipal Ecologic Reserve Selva
\end{abstract}

Trabajo presentado en el marco de la XVI Reunión Argentina de Ornitología (La Plata, 9 al 12 de septiembre de 2015) organizada por la Sección Ornitología, División Zoología Vertebrados, Museo de La Plata, Facultad de Ciencias Naturales y Museo, Universidad Nacional de La Plata. 
Marginal Quilmeña, as well as uncategorized conservation sectors, covering, as a whole, more than 300 hectares. Observations were made by the Bernal's Bird Observers Club, as well as by students from Facultad de Ciencias Naturales y Museo (Universidad Nacional de La Plata). 31 new species were added in comparison to old papers, belonging to the families Tinamidae (1), Falconidae (1), Anatidae (2), Ardeidae (1), Rallidae (3), Strigidae (2), Cuculidae (1), Alcedinidae (1), Furnariidae (7), Sturnidae (1), Motacillidae (1), Thraupidae (2), Tityridae (5), Icteridae (2). Registers on Spartonoica maluroides (Bay-capped Wren-spinetail) and Asthenes hudsoni (Hudson's Canastero) stand out, both of them labeled as vulnerable, besides several other species classified as scarce and sensitive to anthropogenic disturbances. A single specimen of Icterus croconotus (Orange-backed Troupial), a native species from Chaco Húmedo (Wet Chaco) ecoregion, was spotted too. It is noteworthy to point out the recording of Acridotheres cristatellus (Crested Myna), a bird that was introduced in our country and whose impact on native birds and other animal species is unknown. With this contribution, registers with conservationist value were added, and the number of observable species rises from 124 to 155 for this area, which is under an important antropic impact, and it is considered a possible ecological corridor between Punta Lara and Costanera Sur natural reserves.

Key words: Río de la Plata region, biotic corridor, biodiversity, birds, Protected Areas.

\section{Introducción}

Los relevamientos de aves son importantes ya que aportan datos significativos para adoptar medidas en cuanto a la conservación de los ambientes (Alonso et al. 2008; Chebez et al. 1998). Es indispensable saber cuántas aves y en qué abundancia están presentes en un área determinada, para investigar sobre su reproducción, distribución, ecología, entre otros aspectos. También son fundamentales los estudios a largo plazo para entender las variaciones de la diversidad de la avifauna en el tiempo y la influencia que las actividades antrópicas puedan tener sobre ella (Blanco \& Canevari 1993).

En este sentido es que se ha buscado tener un registro completo de la avifauna presente en la costa rioplatense de los partidos de Quilmes y Avellaneda. En estos sitios se encuentran la Reserva Municipal Los Sauces y el Parque Natural y Reserva Ecológica Municipal Selva Marginal Quilmeña, así como también sectores sin categoría de conservación. Para esta área se cuenta con estudios previos recientes sobre avifauna (Godoy et al. 2012) y este trabajo pretende complementar dicho relevamiento, adicionando registros novedosos de aves a las 124 registradas por estos autores. Se busca además, que los resultados aquí expuestos funcionen como una herramienta para la conservación de las zonas estudiadas.

\section{Materiales y métodos}

El área estudiada se encuentra en los partidos de Avellaneda y Quilmes, provincia de Buenos Aires, Argentina, siendo un polígono delimitado por la Autopista Buenos Aires - La Plata y el Canal Santo Domingo (Villa Domínico, partido de Avellaneda) (34²40'52.88"S, 58 $18^{\prime} 55.90^{\prime \prime O}$ ), la desembocadura del Canal Santo Domingo en el Río de La Plata (3440'5.02"S, 58 17'59.20"O), la desembocadura de un arroyo de la localidad de Quilmes en el Río de La Plata (3441'44.72"S, $\left.58^{\circ} 14^{\prime} 48.20^{\prime \prime O}\right)$ y dicho arroyo y la Autopista Buenos Aires - La Plata (3442'19.95"S, $58^{\circ} 15^{\prime} 51.69^{\prime \prime}$ ). Este sector corresponde a la Región Neotropical, Dominio Chaqueño, Provincia Pampeana, Distrito Pampeano Oriental (Cabrera \& Willink 1973). Los ambientes costeros del partido de Quilmes han sido declarados Reserva Municipal bajo las ordenanzas municipales $N^{\circ}$ 8255/96 
(Reserva Natural Municipal Los Sauces) y la $\mathrm{N}^{\circ} 9348 / 02$ y su modificatoria, ordenanza $\mathrm{N}^{\circ} 9508 / 03$ (Parque Natural y Reserva Ecológica Municipal Selva Marginal Quilmeña).

La superficie muestreada posee una extensión de más de 300 hectáreas e incluye una multiplicidad de ambientes: costa, juncal costero, bosque ribereño, eucaliptal, talares, pastizal, pastizal inundable y varios cuerpos de agua que incluyen arroyos, bañados y lagunas (Godoy et al. 2012; Guerrero et al. 2012). En relación a los disturbios antropogénicos se presentan pequeños basurales, proximidad de grandes ciudades al otro lado de la Autopista Buenos Aires-La Plata y pequeños asentamientos hacia el final de la calle Espora (avenida que conecta la ciudad de Bernal con el Río de La Plata) en la costa de Bernal (partido de Quilmes), situaciones de caza y pesca furtiva y fábricas en las cercanías que amenazan la salud de los cuerpos de agua. En cuanto a su relevancia biogeográfica, el sitio constituye el límite austral de distribución de varias especies y es hábitat de animales y plantas poco frecuentes en la región, así como de taxones amenazados por cambios ambientales, todos ellos de filiación paranaense (Guerrero et al. 2012). Además, tiene gran valor como zona de conectividad en la ruta de dispersión biótica Paraná-Uruguay-Plata (Guerrero et al. 2012).

Los relevamientos se realizaron durante sucesivas salidas al campo, comenzando en julio de 2013 y finalizando en enero de 2016, a través de visitas periódicas con un promedio de una visita por mes. Se relevaron los distintos ambientes presentes en el área de estudio, recorriendo senderos, calles, márgenes de arroyos, bañados y lagunas, y las playas de la zona costera. Durante cada jornada de muestreo se recorrieron aproximadamente 5 kilómetros. La avifauna fue muestreada a través de observación directa, utilizando binoculares y mediante registros de canto, por la mañana entre las 8:00 y las 13:00 horas y por la tarde entre las 16:00 y las 20:00 horas. Las tareas se realizaron en conjunto entre alumnos de la Facultad de Ciencias Naturales y Museo, Universidad Nacional de La Plata (FCNyM, UNLP) y el Club de Observadores de Aves de Bernal (COA Bernal).

Se presenta un listado de las especies muestreadas comentando aquellas que poseen valor conservacionista. Para el mismo se siguió el ordenamiento sistemático de Remsen et al. 2016, y se proporcionan los nombres vulgares sugeridos para la Argentina (Navas et al. 1991). Para la identificación de las especies se utilizó guía de campo (Narosky e Yzurieta 2010).

\section{Resultados}

Se contabilizan un total de 155 especies de aves para la zona estudiada (Tabla 1), de las cuales 31 son nuevos registros en el área de estudio (Figura 1) teniendo como referencia el trabajo de Godoy et al. (2012). Tres de los 31 nuevos registros corresponden a especies catalogadas como Vulnerables: Spartonoica maluroides (Espartillero enano), Asthenes hudsoni (Espartillero pampeano) e Icterus croconotus (Matico). Este último, propio de la región chaqueña de Argentina, probablemente represente un ejemplar proveniente de cautiverio, ya que solo observamos un único individuo. Además, se observó una especie introducida: Acridotheres cristatellus (Estornino crestado).

Tabla 1: Lista de aves observadas en la Reserva Municipal Los Sauces y el Parque Natural y Reserva Ecológica Municipal Selva Marginal Quilmeña (partido de Quilmes, provincia de Buenos Aires) y los ambientes costeros del partido de Avellaneda (provincia de Buenos Aires). Se destacan en negrita los 31 nuevos registros para el área aportados por esta contribución.

\begin{tabular}{cccc}
\hline Orden y Familia & Especie & Nombre común & Estatus \\
\hline Tinamiformes & & & NA \\
\hline Tinamidae & Nothura maculosa & Inambú Común & \\
\hline Anseriformes & & & NA \\
\hline Anhimidae & Chauna torquata & Chajá & NA \\
\hline Anatidae & Dendrocygna viduata & Pato sirirí pampa & \\
\hline
\end{tabular}




\begin{tabular}{|c|c|c|c|}
\hline & Heteronetta atricapilla & Pato cabeza negra & $\mathbf{N A}$ \\
\hline & Coscoroba coscoroba & Coscoroba & NA \\
\hline & Cygnus melancoryphus & Cisne cuello negro & NA \\
\hline & Anas sibilatrix & Pato overo & NA \\
\hline & Anas flavirostris & Pato barcino & NA \\
\hline & Anas cyanoptera & Pato colorado & NA \\
\hline & Anas georgica & Pato maicero & NA \\
\hline & Anas platalea & Pato cuchara & NA \\
\hline & Anas versicolor & Pato capuchino & NA \\
\hline & Netta peposaca & Pato picazo & NA \\
\hline & Amazonetta brasiliensis & Pato cutirí & NA \\
\hline & Oxyura vittata & Pato zambullidor chico & NA \\
\hline \multicolumn{4}{|l|}{ Podicipediformes } \\
\hline \multirow[t]{3}{*}{ Podicipedidae } & Rollandia rolland & Macá común & NA \\
\hline & Podilymbus podiceps & Macá pico grueso & NA \\
\hline & Podiceps major & Macá grande & NA \\
\hline \multicolumn{4}{|l|}{ Ciconiiformes } \\
\hline Ciconiidae & Ciconia maguari & Cigüeña americana & NA \\
\hline \multicolumn{4}{|l|}{ Suliformes } \\
\hline Phalacrocoracidae & Phalacrocorax brasilianus & Biguá & NA \\
\hline \multicolumn{4}{|l|}{ Pelecaniformes } \\
\hline \multirow[t]{7}{*}{ Ardeidae } & Butorides striata & Garcita azulada & $\mathbf{N A}$ \\
\hline & Tigrisoma lineatum & Hocó colorado & NA \\
\hline & Nycticorax nycticorax & Garza bruja & NA \\
\hline & Ardea cocoi & Garza mora & NA \\
\hline & Ardea alba & Garza blanca & NA \\
\hline & Egretta thula & Garcita blanca & NA \\
\hline & Syrigma sibilatrix & Chiflón & NA \\
\hline \multirow[t]{3}{*}{ Threskiornithidae } & Plegadis chihi & Cuervillo de cañada & NA \\
\hline & Phimosus infuscatus & Cuervillo cara pelada & NA \\
\hline & Platalea ajaja & Espátula rosada & NA \\
\hline \multicolumn{4}{|l|}{ Accipitriformes } \\
\hline \multirow[t]{3}{*}{ Accipitridae } & Elanus leucurus & Milano blanco & NA \\
\hline & Rupornis magnirostris & Taguató común & NA \\
\hline & Parabuteo unicinctus & Gavilán mixto & NA \\
\hline \multicolumn{4}{|l|}{ Gruiformes } \\
\hline \multirow[t]{8}{*}{ Rallidae } & Gallinula galeata & Pollona negra & $\mathbf{N A}$ \\
\hline & Porphyriops melanops & Pollona pintada & $\mathbf{N A}$ \\
\hline & Laterallus melanophaius & Burrito común & $\mathbf{N A}$ \\
\hline & Aramidea ypecaha & Ipacaá & NA \\
\hline & Aramides cajaneus & Chiricote & NA \\
\hline & Fulica armillata & Gallareta ligas rojas & NA \\
\hline & Fulica leucoptera & Gallareta chica & NA \\
\hline & Fulica rufifrons & Gallareta escudete rojo & NA \\
\hline Aramidae & Aramus guarauna & Carau & NA \\
\hline
\end{tabular}




\begin{tabular}{|c|c|c|c|}
\hline Charadriiformes & & & \\
\hline Charadriidae & Vanellus chilensis & Tero común & NA \\
\hline Recurvirostridae & Himantopus mexicanus & Tero real & NA \\
\hline \multirow[t]{2}{*}{ Scolopacidae } & Tringa melanoleuca & Pitotoy grande & NA \\
\hline & Gallinago paraguaiae & Becasina común & NA \\
\hline Jacanidae & Jacana jacana & Jacana & NA \\
\hline Rostratulidae & Nycticryphes semicollaris & Aguatero & NA \\
\hline \multirow[t]{2}{*}{ Laridae } & Larus dominicanus & Gaviota cocinera & NA \\
\hline & Chroicocephalus maculipennis & Gaviota capucho café & NA \\
\hline Rynchopidae & Rynchops niger & Rayador & NA \\
\hline \multicolumn{4}{|l|}{ Columbiformes } \\
\hline \multirow[t]{5}{*}{ Columbidae } & Columbina picui & Torcacita & NA \\
\hline & Columba livia & Paloma doméstica & I \\
\hline & Patagioenas picazuro & Picazuró & NA \\
\hline & Zenaida auriculata & Torcaza & NA \\
\hline & Leptotila verreauxi & Yerutí común & NA \\
\hline \multicolumn{4}{|l|}{ Psittaciformes } \\
\hline Psittaciformes & Myiopsitta monachus & Cotorra & NA \\
\hline \multicolumn{4}{|l|}{ Cuculiformes } \\
\hline \multirow[t]{2}{*}{ Cuculidae } & Coccyzus melacoryphus & Cuclillo canela & NA \\
\hline & Guira guira & Pirincho & NA \\
\hline Strigiformes & & & NA \\
\hline \multirow[t]{3}{*}{ Strigidae } & Athene cunicularia & Lechucita vizcachera & NA \\
\hline & Pseudoscops clamator & Lechuzón orejudo & $\mathbf{N A}$ \\
\hline & Asio flammeus & Lechuzón de campo & NA \\
\hline \multicolumn{4}{|l|}{ Trochiliformes } \\
\hline \multirow[t]{3}{*}{ Trochilidae } & Chlorostilbon lucidus & Picaflor común & NA \\
\hline & Leucochloris albicollis & Picaflor garganta blanca & NA \\
\hline & Hylocharis chrysura & Picaflor bronceado & NA \\
\hline \multicolumn{4}{|l|}{ Coraciiformes } \\
\hline \multirow[t]{3}{*}{ Alcedinidae } & Chloroceryle amazona & $\begin{array}{c}\text { Martín pescador } \\
\text { mediano }\end{array}$ & NA \\
\hline & Chloroceryle americana & Martín pescador chico & NA \\
\hline & Megaceryle torquata & Martín pescador grande & NA \\
\hline \multicolumn{4}{|l|}{ Piciformes } \\
\hline \multirow[t]{3}{*}{ Picidae } & Colaptes campestris & Carpintero campestre & NA \\
\hline & Colaptes melanochloros & Carpintero real & NA \\
\hline & Veniliornis mixtus & Carpintero bataraz chico & NA \\
\hline \multicolumn{4}{|l|}{ Falconiformes } \\
\hline \multirow[t]{4}{*}{ Falconide } & Falco femoralis & Halcón plomizo & $\mathbf{N A}$ \\
\hline & Falco sparverius & Halconcito colorado & NA \\
\hline & Caracara plancus & Carancho & NA \\
\hline & Milvago chimango & Chimango & NA \\
\hline \multicolumn{4}{|l|}{ Passeriformes } \\
\hline \multirow[t]{2}{*}{ Furnariidae } & Certhiaxis cinnamomeus & Curutié Colorado & NA \\
\hline & Anumbius annumbi & Leñatero & $\mathbf{N A}$ \\
\hline
\end{tabular}




\begin{tabular}{|c|c|c|c|}
\hline & Synallaxis albescens & Pijuí cola parda & NA \\
\hline & Synallaxis spixi & Pijuí plomizo & NA \\
\hline & Synallaxis frontalis & Pijuí frente gris & NA \\
\hline & Phacellodomus striaticollis & $\begin{array}{l}\text { Espinero pecho } \\
\text { manchado }\end{array}$ & NA \\
\hline & Asthenes hudsoni & Espartillero pampeano & $\mathbf{V}$ \\
\hline & Spartonoica maluroides & Espartillero enano & $\mathbf{V}$ \\
\hline & Leptasthenura platensis & Coludito copetón & NA \\
\hline & Lepidocolaptes angustirostris & Chinchero chico & NA \\
\hline & Cinclodes fuscus & Remolinera común & NA \\
\hline & Furnarius rufus & Hornero & NA \\
\hline & Cranioleuca sulphurifera & Curutié ocráceo & NA \\
\hline & Phleocryptes melanops & Junquero & NA \\
\hline \multirow[t]{18}{*}{ Tyrannidae } & Serpophaga griseicapilla & Piojito trinador & NA \\
\hline & Phylloscartes ventralis & Mosqueta común & NA \\
\hline & Myiophobus fasciatus & Mosqueta estriada & NA \\
\hline & Xolmis cinereus & Monjita gris & NA \\
\hline & Xolmis coronatus & Monjita coronada & NA \\
\hline & Xolmis irupero & Monjita blanca & NA \\
\hline & Hymenops perspicillatus & Pico de plata & NA \\
\hline & Tyrannus savana & Tijereta & NA \\
\hline & Tyrannus melancholicus & Suirirí real & NA \\
\hline & Machetornis rixosa & Picabuey & NA \\
\hline & Pitangus sulphuratus & Benteveo & NA \\
\hline & Myiodynastes maculatus & Benteveo rayado & NA \\
\hline & Satrapa icterophrys & Suirirí amarillo & NA \\
\hline & Pyrocephalus rubinus & Churrinche & NA \\
\hline & Elaenia parvirostris & Fio fio pico corto & NA \\
\hline & Pseudocolopteryx flaviventris & Doradito común & NA \\
\hline & Serpophaga nigricans & Piojito gris & NA \\
\hline & Serpophaga subcristata & Piojito común & NA \\
\hline Cotingidae & Phytotoma rutila & Cortarramas & NA \\
\hline Tityridae & Pachyramphus polychopterus & Anambé común & NA \\
\hline \multirow[t]{2}{*}{ Vireonidae } & Vireo olivaceus & Chiví común & NA \\
\hline & Cyclarhis gujanensis & Juan chiviro & NA \\
\hline \multirow[t]{5}{*}{ Hirundinidae } & Tachycineta leucorrhoa & Golondrina ceja blanca & NA \\
\hline & Tachycineta meyeni & Golondrina patagónica & NA \\
\hline & Progne chalybea & Golondrina doméstica & NA \\
\hline & Progne tapera & Golondrina parda & NA \\
\hline & Hirundo rustica & Golondrina tijerita & NA \\
\hline Troglodytidae & Troglodytes aedon & Ratona común & NA \\
\hline Polioptilidae & Polioptila dumicola & Tacuarita azul & NA \\
\hline \multirow[t]{2}{*}{ Turdidae } & Turdus amaurochalinus & Zorzal chalchalero & NA \\
\hline & Turdus rufiventris & Zorzal colorado & NA \\
\hline \multirow[t]{2}{*}{ Mimidae } & Mimus triurus & Calandria real & NA \\
\hline & Mimus saturninus & Calandria grande & NA \\
\hline
\end{tabular}




\begin{tabular}{|c|c|c|c|}
\hline Sturnidae & $\begin{array}{c}\text { Acridotheres cristatellus } \\
\text { Sturnus vulgaris }\end{array}$ & $\begin{array}{c}\text { Estornino crestado } \\
\text { Estornino pinto }\end{array}$ & $\begin{array}{l}\text { I } \\
\text { I }\end{array}$ \\
\hline Motacillidae & Anthus correndera & Cachirla común & $\mathbf{N A}$ \\
\hline \multirow[t]{10}{*}{ Thraupidae } & Donacospiza albifrons & Cachilo canela & $\mathbf{N A}$ \\
\hline & Thraupis sayaca & Celestino común & $\mathbf{N A}$ \\
\hline & Paroaria coronata & Cardenal común & NA \\
\hline & Paroaria capitata & Cardenilla & NA \\
\hline & Sporophila collaris & Corbatita dominó & NA \\
\hline & Sporophila caerulescens & Corbatita común & NA \\
\hline & Sicalis flaveola & Jilguero dorado & NA \\
\hline & Sicalis luteola & Misto & NA \\
\hline & Poospiza nigrorufa & Sietevestidos & NA \\
\hline & Poospiza melanoleuca & Monterita cabeza negra & NA \\
\hline Emberizidae & Zonotrichia capensis & Chingolo & NA \\
\hline Cardinalidae & Pheuticus aureoventris & Rey del bosque & NA \\
\hline \multirow[t]{4}{*}{ Parulidae } & Basileuterus culicivorus & Arañero coronado chico & NA \\
\hline & Myiothlypis leucoblephara & Arañero silbón & NA \\
\hline & Geothlypis aequinoctialis & Arañero cara negra & NA \\
\hline & Setophaga pitiayumi & Pitiayumí & NA \\
\hline \multirow[t]{9}{*}{ Icteridae } & Icterus croconotus & Matico & $\mathbf{V}$ \\
\hline & Icterus cayanensis & Boyerito & NA \\
\hline & Molothrus rufoaxillaris & Tordo pico corto & $\mathbf{N A}$ \\
\hline & Molothrus bonariensis & Tordo renegrido & NA \\
\hline & Cacicus solitarius & Boyero negro & NA \\
\hline & Agelasticus thilius & Varillero ala amarilla & NA \\
\hline & Agelaioides badius & Tordo músico & NA \\
\hline & Sturnella superciliaris & Pecho colorado & NA \\
\hline & Pseudoleistes virescens & Pecho amarillo común & NA \\
\hline \multirow[t]{2}{*}{ Fringillidae } & Spinus magellanicus & Cabecitanegra común & NA \\
\hline & Carduelis chloris & Verderón & I \\
\hline Passeridae & Passer domesticus & Gorrión & $\mathrm{I}$ \\
\hline \multirow[t]{2}{*}{ Incertae sedis } & Saltator aurantiirostris & Pepitero de collar & NA \\
\hline & Saltator coerulescens & Pepitero gris & NA \\
\hline
\end{tabular}

Referencias: NA = No Amenazado, V = Vulnerable, I = Introducido.

\section{Discusión}

En este trabajo se complementa el relevamiento de aves realizado por Godoy et al. (2012) en la Reserva Municipal Los Sauces, el Parque Natural y Reserva Ecológica Municipal Selva Marginal Quilmeña y los ambientes costeros del partido de Avellaneda, adicionando registros novedosos de aves a las 124 registradas por estos autores anteriormente. Se presentan 15 familias de aves pertenecientes a 9 órdenes y se adicionan 31 nuevas especies para el área. Dichos registros elevan el número de especies posibles de observar a 155. La provincia de Buenos Aires posee una variada diversidad de aves, representada por aproximadamente 400 especies (Narosky \& Di Giácomo1993), de modo que gran parte de esa diversidad (38\%) ha sido observada en el área estudiada. 
Se reportan algunas especies que han sido caracterizadas como escasas para el área de estudio (Serpophaga griseicapilla, Bodrati 2006; Xolmis cinereus, López-Lanús et al. 2008) y otras que son consideradas sensibles a los procesos antrópicos de modificación, reemplazo y contaminación del hábitat (Falco femoralis, Heteronetta atricapilla, Porphyriops melanops, Certhiaxis cinnamomeus, Phacellodomus striaticollis, Leptasthenura platensis, Phylloscartes ventralis y Xolmis coronatus; López-Lanús et al. 2008). Por otro lado, Asthenes hudsoni y Spartonoica maluroides presentan importancia en términos de conservación, ya que han sido catalogadas como vulnerables a nivel nacional (López-Lanús et al. 2008), encontrándoselas en menos del 1\% del territorio continental y en menos del 10\% del territorio nacional. Presentan además, una baja amplitud de uso de hábitat, quedando restringidas en sus sitios de residencia a los ambientes de juncal y pajonal. Se reporta también la presencia de Acridotheres cristatellus, especie exótica cuyos primeros registros para la Argentina datan de 1982 para la Reserva de Punta Lara (Moschione 1989). El registro de Icterus croconotus no es habitual para esta área de estudio, ya que es nativo de la ecorregión del Chaco Húmedo y nuestro registro probablemente se trate de un ejemplar proveniente de cautiverio. Sin embargo, Rimoldi \& Pugnali $(2012,2013)$ registraron recientemente una pequeña población en la Reserva Ecológica Costanera Sur, lo que hace pensar que esta especie podría estar prosperando en la ecorregión pampeana.
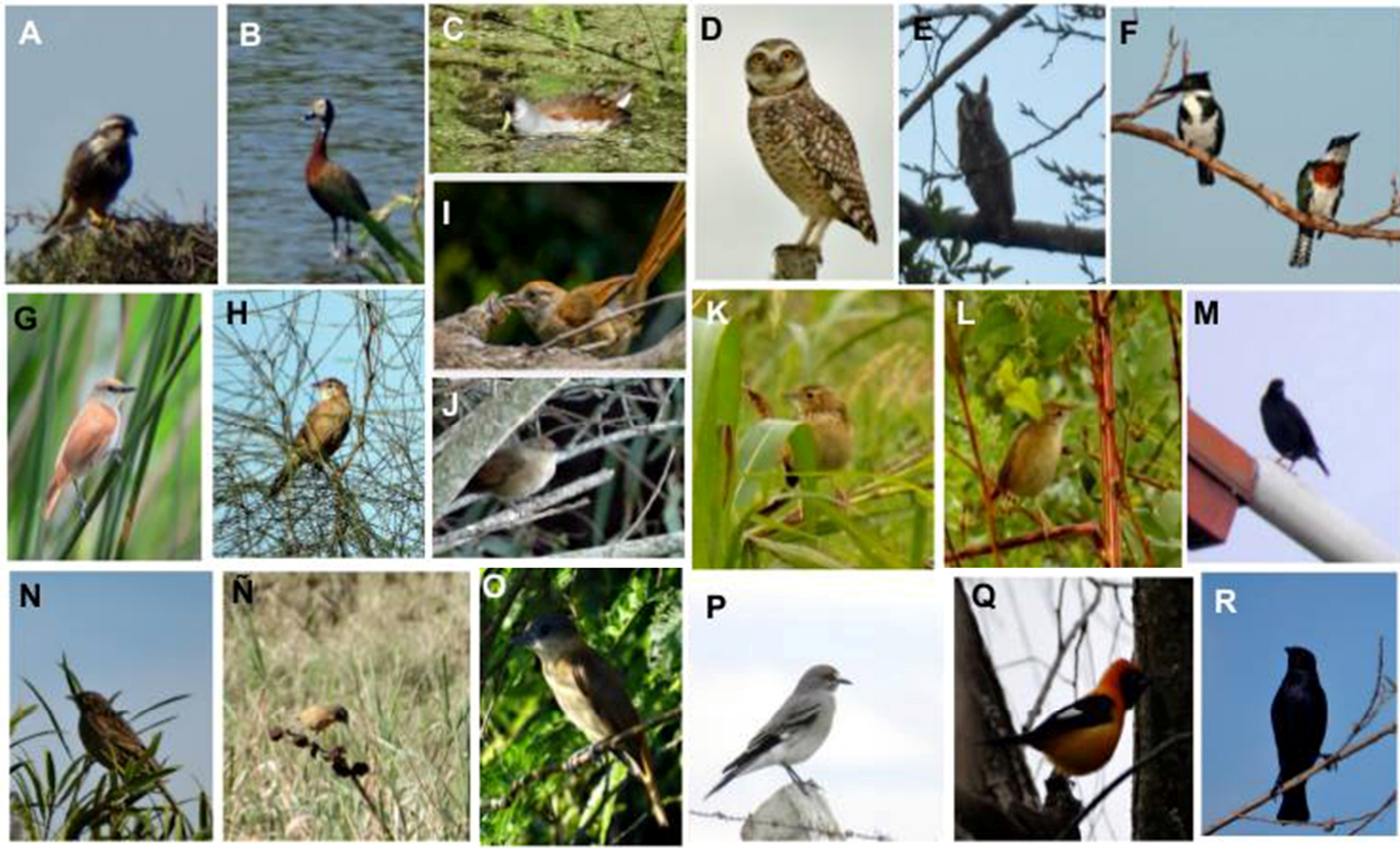

Figura 1. Algunas de las nuevas especies de aves citadas para la zona: A. Falco femoralis; B. Dendrocygna viduata; C. Gallinula melanops; D. Athene cunicularia; E. Pseudoscops clamator; F. Chloroceryle amazona; G. Certhiaxis cinnamomeus; H. Annumbius anumbi; I. Synallaxis albescens; J. Phacellodomus striaticollis; K. Asthenes hudsoni; L. Spartonoica maluroides; M. Acridotheres cristatellus; N. Anthus correndera; N. Donacospiza albifrons; O. Pachyramphus polychopterus; P. Xolmis cinereus; Q. Icterus croconotus; R. Molothrus rufoaxillaris.

Tras la reciente publicación del inventario de aves de la zona costera rioplatense de los partidos de Quilmes y Avellaneda realizado por Godoy et al. (2012), las posteriores visitas al área han dado cuenta de un aumento considerable en el número de especie posibles de observar, entre las que se encuentran algunas con relevancia a nivel conservacionista. Siendo el área de estudio muy cercana a los grandes centros urbanos, el impacto antrópico que esto genera en el ecosistema es muy importante y puede amenazar la diversidad biológica local. Teniendo en cuenta que los bosques ribereños que la 
componen se consideran parte de la ruta de dispersión biótica Paraná-Uruguay-Plata (Guerrero et al. 2012), es relevante la aparición de elementos faunísticos novedosos reportados en este estudio. A modo de conclusión, creemos que contar con datos actualizados de la composición de la avifauna costera rioplatense permite conocer las variaciones cualitativas y cuantitativas en las aves de la zona, a fin de evaluar el impacto antrópico y las consecuencias en el ecosistema. De esta manera, aportamos valiosa información para la toma de decisiones en relación a la conservación de los ambientes involucrados, además de aportar al conocimiento de la dinámica de la zona como ruta de dispersión.

\section{Agradecimientos}

A Mariana Benedictto, Leonel Barral y Natalia Bogado por los aportes en el campo; a Daniel Daglio, Daniela Zaffignani y Martín Colombo por las fotografías cedidas; a Ariel Unamuno, Esteban Niedojadlo y María Florencia Sosa por las traducciones; y a Carolina Acosta Hospitaleche, Federico Agnolin y Diego Isaldo por la revisión de los escritos.

\section{Bibliografía}

Alonso, J. 2008. Inventario de las Aves del Parque Nacional Pre-Delta. Administración de Parques Nacionales. Buenos Aires, Argentina. 96 pp.

Blanco D.E. \& Canevari, P. 1993. Censo Neotropical de Aves Acuáticas 1992. Humedales para las Americas (WA) Buenos Aires, Argentina.

Bodrati, A., Mérida, E., Bodrati, G. \& Sierra, E. 2006. Avifauna del talar de Vuelta de Obligado y de sus ambientes contiguos. San Pedro, provincia de Buenos Aires. En: Mérida, E. y J. Athor (eds.). Talares bonaerenses y su conservación, pp 117124. Fundación de Historia Natural Félix de Azara. Buenos Aires.

Cabrera, A.L. \& Willink, A. 1973. Biogeografía de América Latina. Serie de Biología. Programa Regional Desarrollo Científico y Tecnológico O.E.A., Washington, Monografía 13, 120 pp.

Chebez, J.C., Rey, N., Babarskas, M. \& Di Giacomo, A. 1998. Las Aves de los Parques Nacionales de la Argentina. Monografia Especial L.O.L.A., $N^{o}$ 12, Administración de Parques Nacionales y Asociación Ornitológica del Plata, Buenos Aires, $127 \mathrm{pp}$.

Godoy, I., Suazo Lara, F., Guerrero, E., Rivero, P., Gonzalez, B., Alegre, M., Godoy, A., Kain, C., Sesto, F. \& Chimento N.R. 2012. Relevamiento biótico de la costa rioplatense de los partidos de Quilmes y Avellaneda (Buenos Aires, Argentina). Parte II: Aves. Historia Natural, Tercera Serie, 2(2): 57-94.

Guerrero, E.L., Suazo Lara, F., Chimento, N.R., Buet Constantino, F. \& Simon, P. 2012. Relevamiento biótico de la costa rioplatense de los partidos de Quilmes y Avellaneda (Buenos Aires, Argentina). Parte I: Opiliones (Arachnida), Mygalomorphae (Arachnida), Chilopoda (Miriapoda) y aspectos ambientales destacables. Historia Natural, Tercera Serie 2(2): 31-56.

López-Lanús, B., Grilli, P., Di Giacomo, A.S., Coconier, E.E. \& Banchs, R. 2008. Categorización de las aves de la Argentina según su estado de conservación. Informe de Aves Argentinas/AOP y la Secretaría de Ambiente y Desarrollo Sustentable de la Nación. Buenos Aires, Argentina. 64 pp.

Moschione, F. 1989. Nuevas aves para la Reserva de Punta Lara. Garganchillo 10: 11-12.

Narosky, T. \& Di Giacomo, A.G. 1993. Las aves de la provincia de Buenos Aires. Distribución y estatus. Asociación Ornitológica del Plata. Vázquez Mazini Editores. LOLA. Buenos Aires, Argentina. 127 pp. 
Narosky, T. e Yzurieta, D. 2010. Guía para la Identificación de las Aves de Argentina y Uruguay. Edición total. 16a edición. Vázquez Mazzini Editores. Buenos Aires, Argentina. 432 pp.

Navas, J.R., Narosky, T., Bó, N.A. \& Chebez, J.C. 1991. Lista Patrón de los Nombres Comunes de las Aves Argentinas. Asociación Ornitológica del Plata. Buenos Aires, Argentina. 32 pp.

Remsen, J.V., Jr., Areta, J.I., Cadena, C.D., Jaramillo, A., Nores, M., Pacheco, J.F., Pérez-Emán, J., Robbins, M. B., Stiles, F. G., Stotz, D. F., \& Zimmer, K. J. Version [2016]. A classification of the bird species of South. American Ornithologists' Union. En Línea: http://www.museum.lsu.edu/ Remsen/SACCBaseline.htm.

Rimoldi, C. \& Pugnali, G. 2012. Lista de campo de la Reserva Costanera Sur. Aves registradas 2011/2012. [Fecha de consulta: Septiembre 2015]. Disponible en: http:/www.reservacostanera.com.ar/wp-content/uploads/2013/06/lista-decampo-2011-2012.pdf.

Rimoldi, C. \& Pugnali, G. 2013. Lista de campo de la Reserva Costanera Sur. Aves registradas 2013. [Fecha de consulta: Septiembre 2015]. Disponible en: http://www.reservacostanera.com.ar/wp-content/uploads/2013/06/lista-campo-2013.pdf.

Recibido: noviembre 2015

Aceptado: mayo 2016 